\title{
Optimizing Virtual Machine Placement in Distributed Clouds with M/M/1 Servers ${ }^{\text {th }}$
}

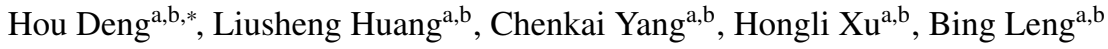 \\ ${ }^{a}$ School of CS \& Tech., Univ. of Science \& Technology of China, Hefei, Anhui 230027, P.R. China \\ ${ }^{b}$ Suzhou Institute for Advanced Study, Univ. of Science \& Technology of China, Suzhou, Jiangsu 215123, P.R. China
}

\begin{abstract}
As more and more applications migrate into clouds, the placement of virtual machines for these applications has a significant impact on the performance of cloud systems. A number of virtual machine (VM) placement techniques have been proposed over recent years. However, most of the existing works on VM placement ignore the response latency of the requests from tenants. In this paper, we investigate the techniques of VM placement in distributed clouds with stochastic requests from the tenants. We first model the requests for each application from the corresponding tenant as independent Poisson stream. Moreover, based on the analyses of distributed cloud resources, the VMs with their data nodes are modeled as simple M/M/1 queueing systems. Then, we propose the problems of VM placement with two distinct optimization objectives. For each objective, we present the formal definition and prove its NP-hardness. To deal with them, we propose some algorithms and the performances of them are analysed in each section. For applying to the situation of lacking of resource, we propose two extended algorithms. We conduct abundant simulation experiments in distributed cloud environment to evaluate the performance of our proposed algorithms. The simulation results show that the proposed algorithms can significantly improve the performance of their corresponding objectives.
\end{abstract}

Keywords: Cloud Systems, Virtual Machine Placement, Stochastic Requests, M/M/1 Queueing System

\section{Introduction}

Cloud computing has emerged in recent years as one of the most interesting developments in technology. The adoption and deployment of cloud computing platforms have many attractive benefits, such as reliability and robustness [1]. As a result, more and more applications are migrating into clouds. Thus, it becomes important for the cloud provider to solve the problem of how to distribute cloud resources efficiently. In modern cloud data centers, e.g. Amazon EC2 [2] and Cisco data center [3], virtual machine (VM) placement is the primary issue facing the effective scheduling of cloud resources [4]. A perfect placement will lead to less cost in resources, such as energy, bandwidth and latency. A number of VM placement techniques have been proposed in the last several years. Xin et al. [5] have introduced a new model, which can be used to guide the design of the resource-balanced VM placement algorithm, and proposed an energy efficient VM placement algorithm EAGLE, which can save about $15 \%$ more energy. O. Biran et al. [6] have dealt with the problem of VM placement in the context of bandwidth usage and network constraints. By considering locations of data centers and system performance constraints, in literature [7], the authors have optimized the VM placement in a geographically distributed cloud environment to minimize the traffic between data centers. Among all these works, VM place-

\footnotetext{
An earlier version of this paper was presented at the 34rd IEEE International Performance Computing and Communications Conference.

${ }^{*}$ Corresponding author.

Email address: dengh@mail.ustc.edu.cn (Hou Deng)
}

ment has played an important role in the resource distribution of cloud system.

As the number of cloud applications increases, there are many popular cloud applications, such as data-intensive applications [8], which are service for dealing with the requests from tenants by data access in cloud systems. Actually, the number of requests from tenants will be large. For example, in Amazon $\mathrm{EC} 2$ [2], the amount of requests is tremendous and growing every day. What's more, the service rate of VM is restricted in data-intensive applications. However, all these works mentioned above ignore the amount of requests and the waiting time of requests in the request queue. Imposing these assumptions leads to vast waiting time of requests in the queue so that the cloud system is inefficient and unreliable, and the user experience becomes poor. In our research, we take the requests from tenants into account and model them as stochastic quantities. The request is typically an aggregation of many independent traffic sources, and by the central limit theorem, it can be approximated using a Poisson random variable [9].

Moreover, the service rate of each VM is restricted and decided by two parts: the rate of processing and the rate of data transmission between VM and its data node when computation and data are spread over a large number of nodes in distributed cloud systems [8]. Since data-intensive applications mainly access data between VMs and their data nodes, we ignore the time of processing. Thus, the service rate of VM is measured by the delay of data transmission between VM and its data $\mathrm{n}$ ode. A request goes to the VM who holds the corresponding application and waits for data transmission in a queue. Therefore, we model each VM with its data node as a queueing sys- 
tem. In this case, the service rate is relevant with the placement of VMs and nodes that store the corresponding data. For instance, if the computation VMs and corresponding data nodes are placed on different places, then the delay of data transmission will be larger and the corresponding service rate will be slower than that placed on the same place. Existing cloud systems, such as Hadoop [10], try to accomplish this by reducing the amount of remote data access. In general, it is impossible to keep most data accesses localized. In this paper, we carefully place computation nodes (VMs) and their data nodes to make data accesses as close as possible, then the service rate will be larger and the response latency will be smaller. Note that the response delay of the request should include the travelling time and waiting time in cloud system. Thus, the distance between tenants and their VMs also be considered in our model. Our contributions in this paper are listed as follows:

1. We model the requests and the VMs with their data nodes as Poisson streams and $\mathrm{M} / \mathrm{M} / 1$ queueing systems respectively, based on which, we define the problems of VM placement with two objectives, including Minimize Maximum Latency (MML) and Minimize Total Latency (MTL). For each problem with a different optimization objective, we formally define it and prove it is NP-hard. To our best knowledge, this is the first work that deals with the problem of VM placement in cloud systems with stochastic requests.

2. For the objective of MML, we propose an approximation algorithm, based on construction of a linear program. We analyse the feasibility and the performance of the proposed algorithm, which can reach the approximate performance of $1+\varepsilon$, where $\varepsilon$ is arbitrary small positive number.

3. For the VM placement problem with MML, we propose three heuristic algorithms, including Greedy, Local Adjustment (LA), and Simulated Annealing (SA). The Greedy heuristic finds VM placement one by one. In order to investigate a better solution, LA heuristic and SA heuristic are proposed based on Greedy heuristic.

4. In the condition of lacking of resource, we guarantee that the number of deployed applications is maximum. We consider the objectives of MML and MTL respectively. Then, two extended algorithms are proposed. which are based on the thought of greed and local search.

5. We conduct abundant simulation experiments in distributed cloud systems to evaluate the performance of our proposed algorithms. The simulation results show that the proposed algorithms are efficient in their corresponding objectives.

The rest of the paper is organized as follows: In Section 2, we discuss the related works. Section 3 describes the system model and problem definition. The detailed solutions of VM placement problem with MML and MTL are given in Section 4 and 5 respectively. The detail extended algorithms are described in Section 6. The simulation results are illustrated in Section 7. We conclude the paper in Section 8.

\section{Related Work}

In this section, we briefly review the related works about VM placement and the optimization of latency in cloud systems.

The technology of VM placement plays an important role in the resource distributed of cloud system. A good VM placement will lead to energy efficiency, low latency, less network cost and more revenue [5],[11], [12], [13]-[22]. As energy consumption increasing in cloud data centers, many researches efforts focus on making the cloud systems more energy efficient in recent years. Gao et al. [13] proposed a multi-objective an$\mathrm{t}$ colony system algorithm for the virtual machine placemen$\mathrm{t}$ problem. Their goal is to efficiently obtain a set of nondominated solutions (the Pareto set) that simultaneously minimize total resource wastage and power consumption. In [14], the authors proposed VMPlanner, a network-wide power manager that optimizes virtual machine (VM) placement and traffic flow routing to reduce data center power costs by sleep scheduling of network elements. Dong et al. [15] proposed a VM placement scheme meeting multiple resource constraints, such as the physical server size and network link capacity to improve resource utilization and reduce both the number of active physical servers and network elements so as to finally reduce energy consumption.

There also exists many studies of VM placement to optimize the latency in cloud systems. In [16], the authors proposed a policy to place the VM with consideration of network conditions to minimize the data transfer time consumption and maintain application performance. To achieve better performance, live migration is adopted in their work. Vu et al. [17] proposed a virtual machine placement mechanism that considers traffic as well as power among VMs within a cloud data center. The goal of this paper is to minimize the communication cost and also save energy. In [18], the authors first modeled a VM placement problem for the total completion time minimization by adopting VM migration, and proposed a migration algorithm that is a heuristic approach. These works do not consider the requests from tenants when place VMs. Different from them, we carefully deploy the VMs to minimize the latency of the requests from tenants.

For the optimization of high throughput, there are also many studies of VM placement. Xin et al. [19] have formulated the VM placement problem for cost minimization, in which both physical machines (PM-cost) and network traffics (N-cost) are taken into account. The problem has been discussed under the three network cost functions, and the authors have presented some algorithms to solve them. Traditionally, VM placement and routing for data center networks are performed separately and the benefits of a joint design are unknown. In [20], the authors have studied a joint tenant (e.g., server or virtual machine) placement and routing problem to minimize traffic costs. Leveraging and expanding the technique of Markov approximation, they have proposed an efficient online algorithm in a dynamic environment under changing traffic loads. For maximizing revenue, Amokrane et al. [21] have proposed Greenhead, a holistic resource management framework for embedding virtual data centers across geographically distributed data centers 


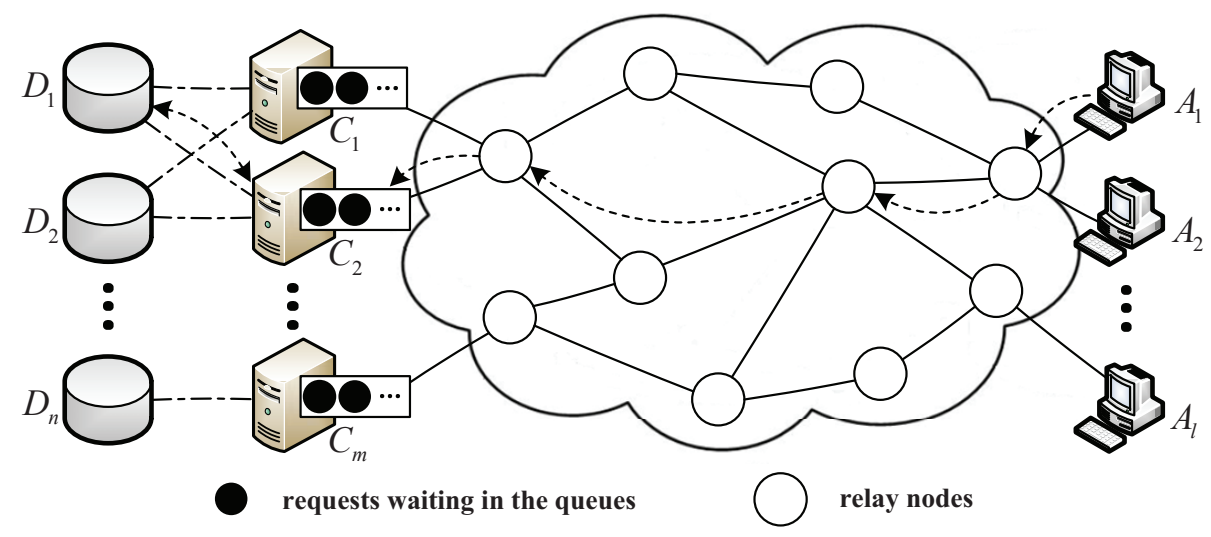

Figure 1: The M/M/1 Queueing System for the Requests from Tenants

connected through a backbone network. They also considered reducing backbone network workload, data center operational costs and the carbon footprint. In literature [22], the authors have proposed a stochastic model to evaluate the performance of migration-enabled cloud systems under an error-prone condition. They implied that a appropriate VM placement plays an important role in preventing migrating VMs.

Recently, as more and more cloud applications benefit from guaranteed latency for their network message, there are many existing works in decreasing the latency in cloud systems [23], [24], [25], [26]. Jang et al. [25] targeted predictable message latency for cloud applications. To achieve this, a general cloud application needs guarantees for its network bandwidth, packet delay and burstiness. They have presented Silo, which enables these guarantees without any network and application changes, relying only on VM placement and end host packet pacing. The services deployed on cloud systems produce large quantities of data continuously. Results from analyzing these geo-distributed data is used by real-time systems and data analysts. In [26], the authors have developed Iridium, a system that focuses on minimizing response times of geo-distributed analytics queries. These studies do not consider the request latency that waiting in the queue. In our study, the VMs with their data nodes are modeled as queueing systems.

\section{System Model}

In this section, we first present the system model. Then, we propose some objectives to optimize the VM placement.

There is a set of tenants denoted by $\left\{A_{1}, A_{2}, \cdots, A_{l}\right\}$. Each of them needs to migrate one data-intensive application into cloud system. In order to provide services to tenants, it is necessary for cloud provider to deploy all applications on the available VMs in the cloud. We assume there are two types of resources: available compute nodes (VMs) and data nodes (DNs) in a cloud data center [8]. Let $\left\{C_{1}, C_{2}, \cdots, C_{m}\right\}, m \geq l$ be the set of available VMs and $\left\{D_{1}, D_{2}, \cdots, D_{n}\right\}, n \geq l$ be the set of available DNs. In addition, we assume one VM accesses data from one DN. For example, in Amazon EC2 environment the compute node mounts the data node as a volume and accesses the volume [8]. We assume one application is processed by one
VM. Moreover, our algorithms can also handle one application processed by multiple VMs by decomposing multiple instance of application.

For the sake of readability, we define two key terminologies of our proposed work.

Definition 1. A resource pair is denoted by a two tuple $\langle j, k>$, where $j$ represents the $V M C_{j}$, and $k$ is the $D N D_{k}$.

Based on the assumption above, each tenant can get, at most, one resource pair to deploy its application in cloud systems.

Definition 2. A tenant assignment is denoted by a three tuple $<i, j, k>$, where $i$ represents the tenant $A_{i}$, and $<j, k>$ is a resource pair.

The link delay of the unit of data transmission between VM $C_{j}$ and DN $D_{k}$ is denoted by $t_{j k}$, and the link delay from tenant $A_{i}$ to VM $C_{j}$ is denoted by $d_{i j}$. Our algorithms can also handle it in geo-distributed clouds by setting the matrixes of $\left[t_{j k}\right]$ and $\left[d_{i j}\right]$. According to the statements in [9], requests could be approximated using a Poisson random variable. We assume that requests from $A_{i}$ form an independent Poisson stream with parameter $\lambda_{i}$. Moreover, the service rate of each VM is limited and decided by data access between $\mathrm{VM}$ and $\mathrm{DN}$. A request goes to a VM and waits for service in a queue. We assume the size of queue is infinite, if not, it may cause packet loss. In order to ensure that the queue does not have too many requests and each request is served in time, we set $\bar{W}_{i}$ to be the upper bound for the average response time of requests from $A_{i}$. Thus, the VMs with their data nodes are modeled as simple $\mathrm{M} / \mathrm{M} / 1$ queueing systems, that is, for each tenant assignment $\langle i, j, k\rangle$, the service time, which is the time of data access between $C_{j}$ and $D_{k}$, at VM $C_{j}$ obeys the exponential distribution with service rate $\mu_{i j k}$. We should notice that the service rate $\mu_{i j k}$ is inversely proportional to the latency $t_{j k}$ and the average data transfer volume $\alpha_{i}$. For simplicity, we assume $\mu_{i j k}=\frac{1}{\alpha_{i} t_{j k}}$. The primary notations used in this paper are summarized in Table I.

The M/M/1 queueing system for the requests from tenants is illustrated in Fig. 1. We assume $C_{2}$ and $D_{1}$ are assigned to $A_{1}$. The request from $A_{1}$ is transmitted to $C_{2}$ via the relay nodes. Because of the limitation of processing capability of $C_{2}$, the request may not be processed immediately, but pushed into the 
Table 1: Primary Notations

\begin{tabular}{|c||c|}
\hline \multicolumn{1}{|c|}{ Symbols } & Descriptions \\
\hline$l$ & Number of the tenants \\
\hline$n$ & Number of the VMs \\
\hline$n$ & Number of the DNs \\
\hline$C$ & Set of the tenants $\left(A=\left\{A_{1}, A_{2}, \cdots, A_{l}\right\}\right)$ \\
\hline$D$ & Set of the VMs $\left(C=\left\{C_{1}, C_{2}, \cdots, C_{m}\right\}\right)$ \\
\hline$d_{i j}$ & Set of the DNs $\left(D=\left\{D_{1}, D_{2}, \cdots, D_{n}\right\}\right)$ \\
\hline$t_{j k}$ & The delay from $A_{i}$ to $C_{j}$ \\
\hline$\lambda_{i}$ & Relay between $C_{j}$ and $D_{k}$ \\
\hline$\mu_{i j k}$ & Service rate for the tenant assignment $<i, j, k>$ \\
\hline $\bar{W}_{i}$ & upper bound for the response time of request from $A_{i}$ \\
\hline$\alpha_{i}$ & The average data transfer volume corresponding to $A_{i}$ \\
\hline
\end{tabular}

waiting queue. When processing it, the $\mathrm{VM} C_{2}$ will access data with $D_{1}$. Thus, the response delay for a request consists of the link delay and the waiting time in cloud system.

There are several possible objectives which optimize the VM placement. We can use any of the following objectives while deploying the application:

A. Minimize Maximum Latency (MML): The cloud provider wants to decrease the maximum latency for the tenants to improve user experience. In this case, our goal is to minimize the maximum response latency of the requests from tenants. We assume there are enough cloud resources, and all applications will be deployed on cloud systems. The solution may lead to very high total latency.

B. Minimize Total Latency (MTL): In order to reduce the overall system cost (i.e. power, time etc), the cloud provider wants to decrease the overall response latency. In this case, we minimize the sum of the average latency of the requests from tenants. We also assume there are enough cloud resources, and all applications will be deployed on cloud systems. The solution may lead to very high minimum latency.

We can see that the above objectives are under the assumption that there are enough cloud resources. However, the cloud data center may have not enough resource, not all of the tenants can get the resources [27]. In this paper, we also take it into consideration. When the cloud system is deficient in resources, the cloud provider will want to provide as many tenants as possible with could resources to maximize the benefits, that is, the Maximum Number of deployed Applications (MNA) needs to be insured. In this case, we also consider the objectives of MML and MTL, respectively.

\section{Solution of MML}

In this section, our goal is to find a VM placement such that the maximum response latency of the requests is minimum. We formulate the problem with the objective of MML, and prove it is NP-hard. Then, we propose an approximation algorithm. We also analyse the feasibility and the approximate performance of the proposed algorithm.

\subsection{Problem Formulation}

A binary three-dimensional matrix $x=\left[x_{i j k}\right]$ is introduced for it, which is assumed as:

$$
x_{i j k}= \begin{cases}1, & \text { if the resource } C_{j} \text { and } D_{k} \text { is assigned to } A_{i}, \\ 0, & \text { otherwise, }\end{cases}
$$

where $\forall A_{i} \in A, \forall C_{j} \in C, \forall D_{k} \in D$.

We formulate the constraint of our problem. All applications are deployed on cloud system, it obeys the following constraint:

$$
\sum_{j: C_{j} \in C} \sum_{k: D_{k} \in D} x_{i j k}=1, \quad \forall i: A_{i} \in A .
$$

Since each resource will be assigned to only one tenant at most, the formulation can be written as:

$$
\begin{aligned}
& \sum_{i: A_{i} \in A} \sum_{k: D_{k} \in D} x_{i j k} \leq 1, \quad \forall j: C_{j} \in C . \\
& \sum_{i: A_{i} \in A} \sum_{j: C_{j} \in C} x_{i j k} \leq 1, \quad \forall k: D_{k} \in D .
\end{aligned}
$$

Next, in order to get the constraints of the latency demands of $\bar{W}_{i}$, we first formulate the response delay, which is the period of time from the beginning of a request to the end of its processing. The response delay can be divided into two parts: the travelling time and waiting time. The aggregate travelling time of requests from the tenant $A_{i}$ per unit time is

$$
P_{i}=\sum_{j: C_{j} \in C} \sum_{k: D_{k} \in D} \lambda_{i} d_{i j} x_{i j k}
$$

Since each VM with its data node behaves as an M/M/1 queueing system, when the resource $C_{j}$ and $D_{k}$ is assigned to tenant $A_{i}$, the expected waiting time at $C_{j}$ is $W_{i j k}=1 /\left(\frac{1}{\alpha_{i} t_{j k}}-\lambda_{i}\right)$ [28]. Thus, the aggregate waiting time of requests from the tenant $A_{i}$ per unit time is

$$
Q_{i}=\sum_{j: C_{j} \in C} \sum_{k: D_{k} \in D} \lambda_{i} x_{i j k} W_{i j k} .
$$

Thus, the aggregate response delay is $P_{i}+Q_{i}$, and the constraint can be written as:

$$
\sum_{j: C_{j} \in C} \sum_{k: D_{k} \in D} x_{i j k} d_{i j k}^{\prime} \leq \bar{W}_{i}, \quad \forall i: A_{i} \in A
$$

where $d_{i j k}^{\prime}=\lambda_{i}\left(d_{i j}+W_{i j k}\right)$ represents the aggregate response time of requests of the tenant assignment $\langle i, j, k>$.

Finally, the parameters can be formally defined as:

$$
x_{i j k} \in\{0,1\}, \quad \forall i: A_{i} \in A, \forall j: C_{j} \in C, \forall k: D_{k} \in D .
$$

the VM placement problem with the objective of MML is presented as:

$$
\text { Minimize } \max _{i: A_{i} \in A} \sum_{j: C_{j} \in C} \sum_{k: D_{k} \in D} x_{i j k} d_{i j k}^{\prime}, \quad \text { subject to: (1)-(5). }
$$

As shown above formulation, the main factors that lead to the hardness of this problem include the large number of constraints and the binary nature of the decision variables. It is proved that the VM placement problem with the objective of MML is NPhard in the following theorem. 
Theorem 1. The VM placement problem with MML is an NPhard problem.

Proof: As a decision problem, we ask simply is there a VM placement with the maximum latency $T$ in the cloud systems. Let A, C, D be three disjoint set, and $E$ be the subset of $A \times C \times D$. If and only if $d_{i j k}^{\prime} \leq \min \left\{\bar{W}_{i}, T\right\}$, the triple $<i, j, k>\in E$. our problem is to find a subset $\bar{E} \subseteq E$ such that $|\bar{E}|=|A|$ and for any two distinct triples $<i_{1}, j_{1}, k_{1}>\in \bar{E}$ and $<i_{2}, j_{2}, k_{2}>\in \bar{E}$, we have $i_{1} \neq i_{2}, j_{1} \neq j_{2}$ and $k_{1} \neq k_{2}$. So far, This decision problem was also reduced to the Max 3-dimensional matching problem [29], which is NP-hard. Thus, the VM placement problem with MML is NP-hard.

\subsection{Algorithm Description}

In this section, we describe an approximate algorithm of VM placement problem with the objective of MML. According to the above proof of NP-hardness, our problem can be reduced to the Max 3-dimensional matching problem. As shown in the literature [30], to solve the Max 3-dimensional matching problem, the author have proposed a $(3 / 2+\varepsilon)$-approximation algorithm. To improve the approximation ratio for this problem, Cygan [31] has proposed an algorithm that can reach the approximate performance of $(4 / 3+\varepsilon)$. However, in this paper, to solve our problem, we propose a polynomial-time algorithm, which can reach the approximate performance of $(1+\varepsilon)$, where $\varepsilon$ is arbitrary small positive number. In order to simplify the objective function, it can be transformed to:

Minimize $T$ where $T$ is that:

$$
\sum_{j: C_{j} \in C} \sum_{k: D_{k} \in D} x_{i j k} d_{i j k}^{\prime} \leq T, \quad \forall i: A_{i} \in A .
$$

The algorithm consists of three steps, initialization, binary search and resource assignment, respectively. At the beginning, all parameters including $d_{i j k}^{\prime}$ are initialized. In order to minimize the maximum latency $T$, our algorithm uses the binary search method. Thus, two variables $T h$ and $T l$ need to be initialized, where $T h$ is the upper bound of $T$ and $T l$ is the lower bound of $T$. It is known that $T$ is less than $\max \left\{d_{i j k}^{\prime}\right\}$ and more than $\min \left\{d_{i j k}^{\prime}\right\}$. To deploy all applications, $T$ is also less than $\min \left\{\bar{W}_{i}\right\}$. We can obtain that $T h=\min \left\{\max \left\{d_{i j k}^{\prime}\right\}, \min \left\{\bar{W}_{i}\right\}\right\}$, $T l=\min \left\{d_{i j k}^{\prime}\right\}$.

After the initialization, the process enters the step of binary search. From the constraint (7), it is known that:

$$
\forall A_{i} \in A, \forall C_{j} \in C, \forall D_{k} \in D \text {, if } d_{i j k}^{\prime}>T \text { then } x_{i j k}=0
$$

However, this is not a linear constraint. We will use the technique of parametric pruning to get around this difficulty. At each iteration, let $T=\frac{T h+T l}{2}$, which enables us to prune away all tenant assignments $\left\langle i, j, k>\right.$ such that $d_{i j k}^{\prime}>T$. Define $S(T)=\left\{<i, j, k>\mid d_{i j k}^{\prime} \leq T\right\}$. We can construct a linear pro-

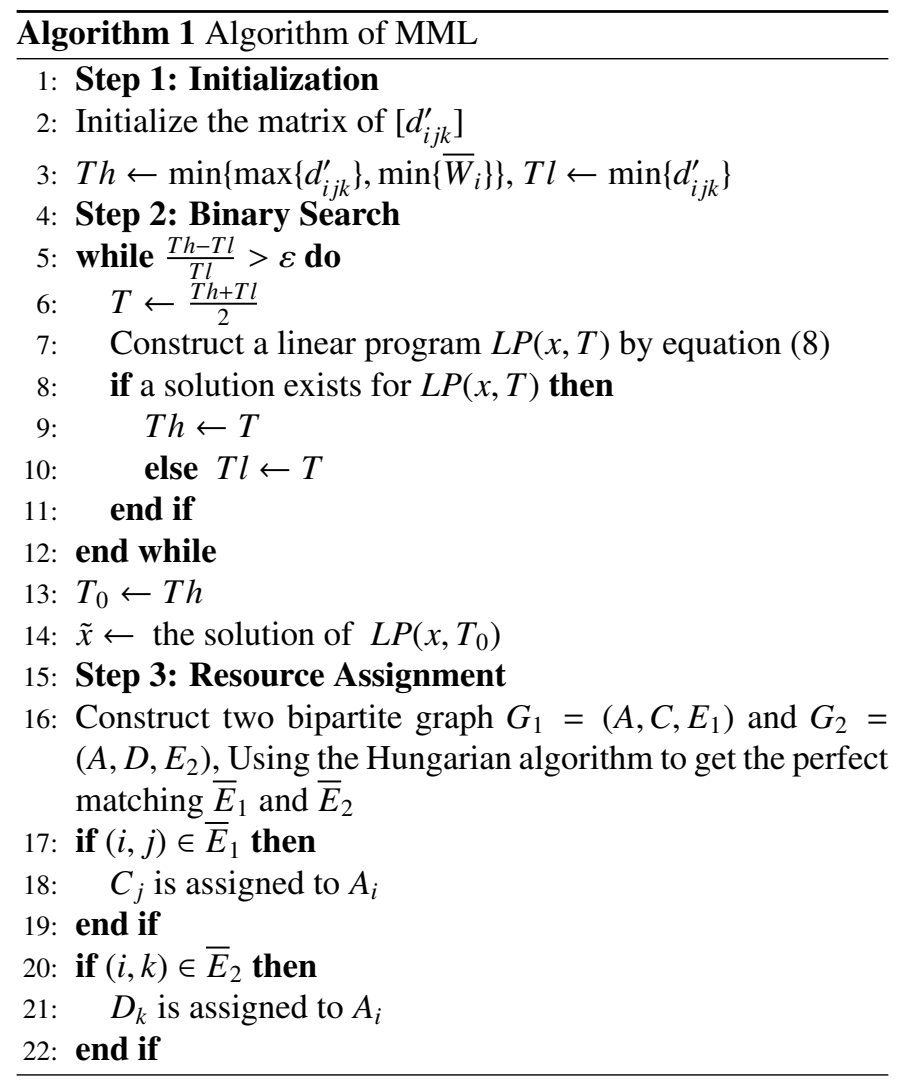

gram $L P(x, T)$ for a given parameter $T$ :

$$
\begin{gathered}
\text { S.t. } \quad \sum_{j, k:<i, j, k>\in S(T)} x_{i j k}=1, \forall i: A_{i} \in A, \\
\sum_{i, k:<i, j, k>\in S(T)} x_{i j k} \leq 1, \forall j: C_{j} \in C, \\
\sum_{i, j:<i, j, k>\in S(T)} x_{i j k} \leq 1, \forall k: D_{k} \in D, \\
\sum_{j, k:<i, j, k>\in S(T)} x_{i j k} d_{i j k}^{\prime} \leq T, \forall i: A_{i} \in A, \\
x_{i j k} \in[0,1], \forall<i, j, k>\in S(T) .
\end{gathered}
$$

If there is a solution for the linear program $L P(x, T)$, this means that the minimum latency $T$ is an approximate upperbound for the VM placement problem. So we set $T h=T$. Otherwise, no solution is found, which means that the minimum latency $T$ can not be reached by the resources assigning, so $T l=T$. This iterative process will be terminated if $\frac{T h-T l}{T l} \leq \varepsilon$, where $\varepsilon$ is arbitrary small positive number. At the end of this step, we regard that $T h$ is an approximate upper-bound, denoted by $T_{0}$, for VM placement. The optimal solution of $\operatorname{LP}\left(x, T_{0}\right)$, denoted by $\tilde{\mathrm{x}}$, can be derived.

The third step is implementing the resource assignment. According to an optimal solution $\tilde{\mathrm{x}}$ of $L P\left(x, T_{0}\right)$, we define $G_{1}=$ $\left(A, C, E_{1}\right)$ to be the bipartite graph on vertex set $A \cup C$, where $(i, j) \in E_{1}$ if and only if $x_{i j k} \neq 0$, and define $G_{2}=\left(A, D, E_{2}\right)$ to be the bipartite graph on vertex set $A \cup D$, where $(i, k) \in E_{2}$ if and only if $x_{i j k} \neq 0$. It can be obtained the perfect matchings $\bar{E}_{1}$ and $\bar{E}_{2}$ of the bipartite graphs $G_{1}$ and $G_{2}$ respectively by 


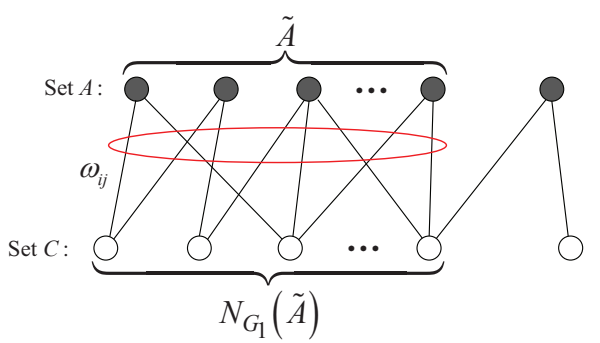

Figure 2: The bipartite graph of $G_{1}$

using Hungarian algorithm [32]. The existence of the perfect matching is proved by Theorem 3. Then, we can get the resource assignment that ensure the maximum delay is less than $T_{0}$. if $(i, j) \in \bar{E}_{1}$, the VM $C_{j}$ is assigned to the tenant $A_{i}$, and if $(i, k) \in \bar{E}_{2}$ the DN $D_{k}$ is assigned to the tenant $A_{i}$. The detailed algorithm description is shown in Alg. 1.

\subsection{Feasibility and Performance}

We first prove the following theorem to ensure that the above algorithm can get a solution of the VM placement problem.

Theorem 2. Both bipartite graphs $G_{1}$ and $G_{2}$ used in Alg. 1 exist the perfect matching.

Proof: We first prove that the bipartite graph $G_{1}=\left(A, C, E_{1}\right)$ exists perfect matching by using the Hall Theorem [33]. The Hall Theorem can be described as follows: Let $G$ be a finite bipartite graph with bipartite sets $X$ and $Y$, there is a matching that entirely covers $X$ if and only if $\left|N_{G}(\widetilde{X})\right| \geq|\widetilde{X}|, \forall \widetilde{X} \subseteq X$, where $N_{G}(\widetilde{X})$ is the set of the neighborhood of $\widetilde{X}$ in $G$. Thus, we only need to prove that $\left|N_{G_{1}}(\widetilde{A})\right| \geq|\widetilde{A}|, \forall \widetilde{A} \subseteq A$. Suppose that this is not the case, that is $\exists \widetilde{A} \subseteq A,\left|N_{G_{1}}(\widetilde{A})\right|<|\widetilde{A}|$, which is shown in Fig. 2. In this figure, the black nodes and the white nodes represent the set $A$ and the set $C$, respectively. For each edge $(i, j), \forall A_{i} \in \widetilde{A}, \forall C_{j} \in N_{G_{1}}(\widetilde{A})$, which is circled by a red circle in the figure, we give a weight $\omega_{i j}=\sum_{k:<i, j, k>\in S\left(T_{0}\right)} x_{i j k}$. The sum of these weights $\mathbb{S}$ can be represented as:

$$
\mathbb{S}=\sum_{A_{i} \in \widetilde{A}} \sum_{j:(i, j) \in E_{1}} \omega_{i j}
$$

According to the equation ( $8 \mathrm{a})$, it can be calculated that:

$$
\begin{aligned}
\mathbb{S} & =\sum_{A_{i} \in \widetilde{A}} \sum_{C_{j} \in N_{G_{1}}(\widetilde{A})} \sum_{k:<i, j, k>\in S\left(T_{0}\right)} x_{i j k} \sum_{A_{i} \in \widetilde{A}} \sum_{j:<i, j, k>\in S\left(T_{0}\right)} x_{k:<i, j, k>\in S\left(T_{0}\right)} \\
& =\sum_{A_{i} \in \widetilde{A}} 1=|\widetilde{A}| .
\end{aligned}
$$

On the other hand, the inequality (8b) shows that:

$$
\begin{aligned}
\mathbb{S} & =\sum_{C_{j} \in N_{G_{1}}(\widetilde{A})} \sum_{A_{i} \in \widetilde{A}} \sum_{k:<i, j, k>\in S\left(T_{0}\right)} x_{i j k} \sum_{C_{j} \in N_{G_{1}}(\widetilde{A})} \sum_{i:<i, j, k>\in S\left(T_{0}\right)} \sum_{k:<i, j, k>\in S\left(T_{0}\right)} x_{i j k} \\
& \leq \sum_{C_{j} \in N_{G_{1}}(\widetilde{A})} 1=\left|N_{G 1}(\widetilde{A})\right|
\end{aligned}
$$

Thus, $|\widetilde{A}| \leq\left|N_{G_{1}}(\widetilde{A})\right|$, leading to a contradiction. In conclusion, the bipartite graph $G_{1}$ exists perfect matching. Similarly, the bipartite graph $G_{2}$ exists perfect matching by using the same method. The theorem is proved.

After the second step, the algorithm obtains the approximate upper-bound $T_{0}$. We first prove the following lemma.

Lemma 3. Assume that the optimal result for the VM placement problem with MML is $T_{\text {opt }}$. It follows that $T_{0} \leq(1+\varepsilon) T_{\text {opt }}$.

Proof: Under the delay constraint $T_{o p t}$, there must exist a solution for the linear program $L P\left(x, T_{\text {opt }}\right)$. Since there is no solution for $L P(x, T l)$, it can be obtained that $T_{o p t} \geq T l$. At the end of the second step, $T_{0}=T h$. Thus,

$$
\frac{T_{0}}{T_{o p t}} \leq \frac{T_{0}}{T l}=\frac{T h}{T l} \leq 1+\varepsilon
$$

This indicates $T_{0} \leq(1+\varepsilon) T_{o p t}$. The lemma is proved.

Theorem 4. The algorithm of MML can reach the approximate performance of $1+\varepsilon$ for the VM placement problem with $M M L$.

Proof: After the second step, the algorithm determines an approximate upper-bound, denoted by $T_{0}$, for VM placemen$\mathrm{t}$ problem with MML. Then, we will find a solution, denoted by $\tilde{\mathrm{x}}$, for the linear program $L P\left(x, T_{0}\right)$. By constructing two bipartite graph $G_{1}$ and $G_{2}$ and using the Hungarian algorithm respectively, we can find the perfect matching $\bar{E}_{1}$ and $\bar{E}_{2}$, corresponding to two types of resources assignment. Let $T_{M M L}$ be the final result of the algorithm of MML. It can be derived $T_{M M L} \leq T_{0}$, because of the construction of $S\left(T_{0}\right)$. Thus,

$$
\frac{T_{M M L}}{T_{o p t}} \leq \frac{T_{0}}{T_{o p t}} \leq 1+\varepsilon
$$

So far, we proved that the algorithm of MML can reach the approximate performance of $1+\varepsilon$.

Since $T_{\text {opt }}$ is the optimal solution of the VM placement with MML, it can be known that $T_{o p t} \leq T_{M M L} \leq T_{h}$. We can obtain that:

$$
\frac{T_{M M L}}{T_{o p t}} \geq \frac{T_{M M L}}{T_{h}} \geq \frac{T_{l}}{T_{h}} \geq \frac{1}{1+\varepsilon} .
$$

Thus, when $\varepsilon$ is infinitely closed to 0 , our solution is optimal.

\section{Solutions of MTL}

In this section, we aim to solve the VM placement problem with the objective of MTL. We formulate it, and prove it is NP-hard. Then, we propose three heuristic algorithms, including Greedy, Local Adjustment (LA), and Simulated Annealing (SA). 


\subsection{Problem Formulation}

Based on the analysis of the constraints in Section 4.1, the VM placement problem with MTL is presented as:

$$
\min \sum_{i: A_{i} \in A} \sum_{j: C_{j} \in C} \sum_{k: D_{k} \in D} x_{i j k} d_{i j k}^{\prime}, \quad \text { subject to: (1)-(5). }
$$

Theorem 5. The VM placement problem with MTL is an NPhard problem.

Proof: We assume the number of tenants, VMs and DNs are the same, that is, $l=m=n$, and the upper bound for the response time of the requests $\bar{W}_{i}$ is infinity. In this case, all of the resources including VMs and DNs are assigned to tenants. Thus, the constraints (2), (3) will be equal. The constraint (4) can be ignored. The original problem is reduced to axial 3-dimensional assignment problem (3-DAP), and Karp [34] showed that the axial 3-DAP is NP-hard. So the VMMD is an NP-hard problem.

\subsection{Algorithms Description}

We tackle the problem by using heuristics. In this section, three heuristic algorithms are developed for the problem. The first one is the Greedy heuristic. The second is an improvement of the first one using Local Adjustment heuristic. Thirdly, a global searching method simulated annealing heuristic will be proposed.

\section{A) Greedy Algorithm}

Firstly, we introduce a greedy heuristic algorithm. Generally speaking, the greedy strategy is often used to solve the optimization problem. In the VM placement problem with MTL, there are something important to consider. First, in our constraint, it should ensure that the response time for the requests from $A_{i}$ does not exceed $\bar{W}_{i}$, and we solve it by assuming the delay of the tenant assignment is $\Gamma$ when the response time exceed $\bar{W}_{i}$, where $\Gamma$ is a large enough positive number. Then, it can be noticed that each resource, including the VMs and the DNs, can only be assigned to one tenant. Thus, in our algorith$\mathrm{m}$ we need to remove the allocated resources in each iteration.

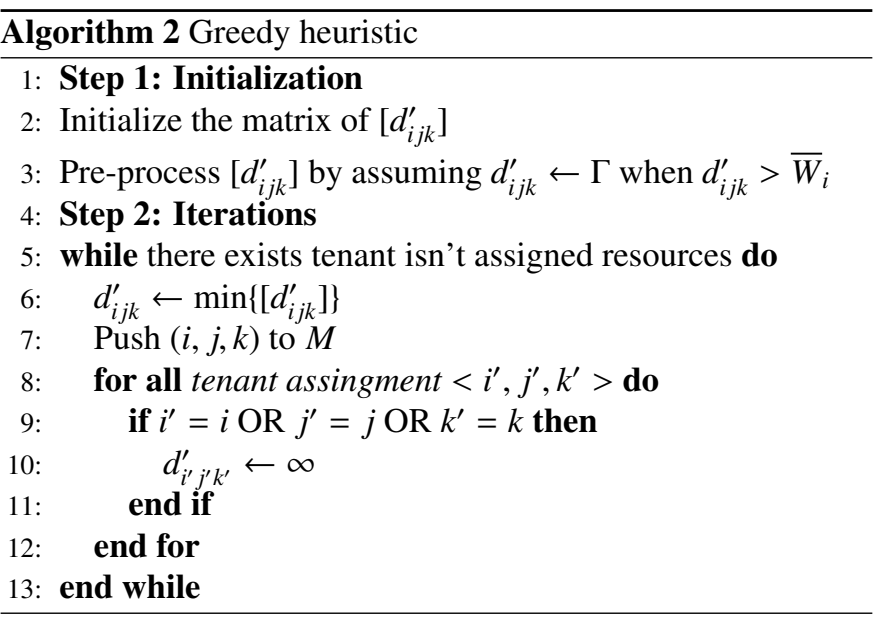

The system parameter in Table. I will be initialized in the beginning of Greedy heuristic. According to the method above, the delay matrix of $d_{i j k}^{\prime}$ could be obtained. In each iteration, we find a tenant assignment $\left\langle i, j, k>\right.$ so that the delay $d_{i j k}^{\prime}$ is minimum in the matrix $\left[d_{i j k}^{\prime}\right]$, and we assign the resources $C_{j}$ and $D_{k}$ to $A_{i}$. Afterwards, the tenant $A_{i}$ and the allocated resources $C_{j}$ and $D_{k}$ should be removed by setting the element that is related to $A_{i}, C_{j}$ and $D_{k}$ in the matrix $\left[d_{i j k}^{\prime}\right]$ to infinity. The iteration is terminated when all of the tenants are assigned resources. The detailed algorithm description is shown in Alg. 2.

\section{B) Local Adjustment Algorithm}

Because of the limitation of the service rate of the VM, the Greedy heuristic algorithm may find an infeasible solution, in which there exists an application that the response time of its corresponding requests exceeds $\bar{W}_{i}$. In order to investigate a better solution, or to possibly find feasible solution in the case of Greedy heuristic fails, we propose Local Adjustment (LA) heuristic that takes the relation between two applications into account. In order to easily introduce this algorithm, we define one key terminology of our proposed work.

Definition 3. given a solution, assume arbitrary two tenants $A_{i}$ and $A_{i^{\prime}}$ are assigned the resource pair $<j, k>$ and $<$ $j^{\prime}, k^{\prime}>$ respectively. The Adjustable Set of the tenant $A_{i}$ and $A_{i^{\prime}}$ is divided into two parts: $C^{\prime}\left(i, i^{\prime}\right)$ and $D^{\prime}\left(i, i^{\prime}\right)$, where $C^{\prime}\left(i, i^{\prime}\right)=\left\{j, j^{\prime}\right\} \cup\left\{j^{\prime \prime} \mid C_{j^{\prime \prime}} \in C \& \& C_{j^{\prime \prime}}\right.$ isn't assigned to any tenant in this solution $\}$ is the VM Adjustable Set. $D^{\prime}\left(i, i^{\prime}\right)=$ $\left\{k, k^{\prime}\right\} \bigcup\left\{k^{\prime \prime} \mid D_{k^{\prime \prime}} \in D \& \& D_{k^{\prime \prime}}\right.$ isn't assigned to any tenant in this solution is the DN Adjustable Set.

For example, in Fig. 1, we assume $l=3, m=4, n=5$, and a solution $\{<1,1,1>,<2,2,2>,<3,3,3>\}$. the adjustable set of $A_{1}$ and $A_{2}$ is $C^{\prime}(1,2)=\{1,2,4\}$ and $D^{\prime}(1,2)=\{1,2,4,5\}$. Clearly, the tenant $A_{i}$ and $A_{i^{\prime}}$ can select resources from $C^{\prime}\left(i, i^{\prime}\right)$ and $D^{\prime}\left(i, i^{\prime}\right)$ without affecting on other tenants. The LA heuristic starts with a random solution. Since the Greedy algorithm is a convenient way to generate the initial solution, it is applied first. Then, LA algorithm adjusts the assignment with the goal of decreasing the objective function in each iteration. Note that in order to adjust the assignment, it is necessary to solve the problem that how to find the adjustable tenant assignments. In our algorithm, we consider the relation between two applications. If there exists a tenant pair that makes the objective decrease by adjusting their assignments without affecting on other tenants, we can reallocate the resources to this two tenants. In order to judge weather there exists the adjustable tenant pair, we traverse all of the tenant pairs. For each tenant pair $\left(i, i^{\prime}\right)$, their adjustable set $C^{\prime}\left(i, i^{\prime}\right)$ and $D^{\prime}\left(i, i^{\prime}\right)$ can be obtained according to Definition 4. Then, we figure out a pair of resource pair that meet the following conditions:

1. They are in the adjustable set $C^{\prime}\left(i, i^{\prime}\right)$ and $D^{\prime}\left(i, i^{\prime}\right)$;

2. They are not the same resource;

3. When they are assigned to $A_{i}$ and $A_{i^{\prime}}$ respectively, the sum of their delay denoted by $s$ should be minimum among all 


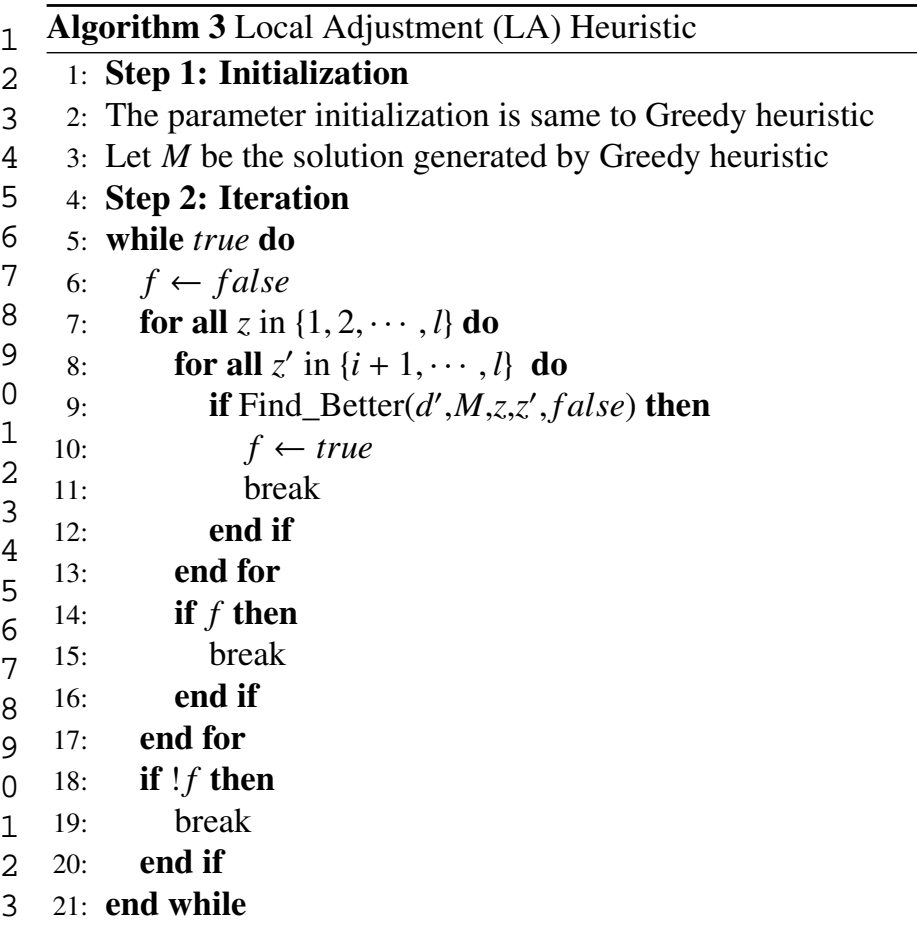

If $s$ is less than the sum of delay of $A_{i}$ and $A_{i^{\prime}}$ in current solution, that is, $\left(i, i^{\prime}\right)$ is an adjustable tenant pair, and we reallocate this pair of resource pair to them. Otherwise, we test other tenant pairs. The iteration traverse all of the tenant pairs to find an adjustable tenant pair until there doesn't exist it. Once an adjustable tenant pair is found, it is reallocated, and the algorithm enter the next iteration. The detailed description of LA heuristic is shown in Alg. 3. The Find_Better sub-procedure used in LA heuristic is described in Alg. 4, in which the parameter $S A$ is false.

\section{C) Simulated Annealing Algorithm}

The solutions obtained from Greedy heuristic and LA heuristic are local optimization. Based on LA heuristic, we propose Simulated Annealing(SA) heuristic that is a global searching algorithm in theory. It is necessary to find an initial solution for SA heuristic. we can use the solution obtained from LA heuristic. Different from traditional simulated annealing, we use a global parameter $M$ to record the best solution that is found in the second step. We can initialize this parameter by the initial solution. In each iteration, the parameter $M$ is updated to the solution if it's better.

After an initial solution is obtained, the iteration starts. Based on the searching method in LA heuristic, the searching neighborhood $\mathcal{N}$ is defined as all of the tenant pairs. In each iteration, we randomly test $\Omega_{2}$ tenant pairs from $\mathcal{N}$, where $\Omega_{2}$ is a positive integer defined in advance. For each tenant pair, we identify it as an adjustable tenant pair by the method from LA heuristic. If it is an adjustable tenant pair, the SA heuristic perform the

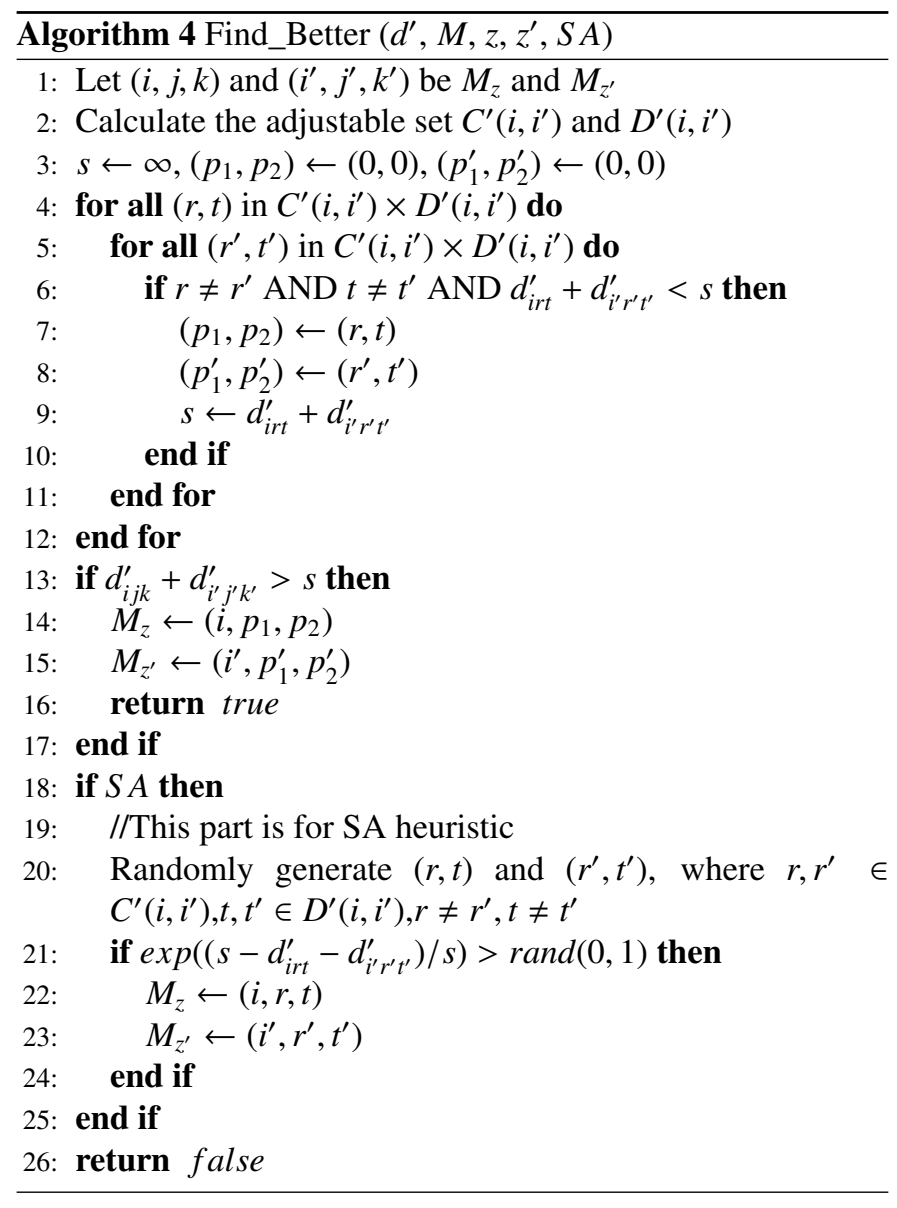

same way as LA heuristic to reallocate the resources to this pair of tenant, and terminates the test for other tenant pairs. When there doesn't exist the adjustable one in these $\Omega_{2}$ tenant pairs, we randomly select an tenant pair $\left(z, z^{\prime}\right)$ from $\mathcal{N}$, and probabilistically decide whether reallocate resources to them. If $\left(z, z^{\prime}\right)$ is an adjustable pair, the probability is 1 to reallocate the pair of resource pair obtained by the way of LA heuristic to them. Otherwise, a pair of resource pair without the same resource from the adjustable set of $\left(z, z^{\prime}\right)$ should be generated randomly. For this pair of resource pair, we let $s^{\prime}$ be the sum of the delay when it is assigned to $\left(z, z^{\prime}\right)$. The probability is $\exp (-\Delta / s)$ to reallocate this pair of resource pair to $\left(z, z^{\prime}\right)$, where $s$ is the sum of delay of $\left(z, z^{\prime}\right)$ in current solution, and $\Delta=s^{\prime}-s$. The iteration is terminated until one of the following stopping conditions is met:

1. The number of iterations is greater than $\Omega_{1}$, where $\Omega_{1}$ is a predefined positive integer.

2. They do not exist the adjustable tenant pair in $\Omega_{3}$ consecutive iterations, where $\Omega_{3}$ is a predefined positive integer.

At the end of the iteration, the parameter $M$ is the assignment we need. The detailed description of SA heuristic is shown in Alg. 5. The Find_Better sub-procedure used in SA heuristic is described in Alg. 4.

Since the Greedy heuristic is a sub-procedure of the LA heuristic, which is a sub-procedure of the SA heuristic, the re- 


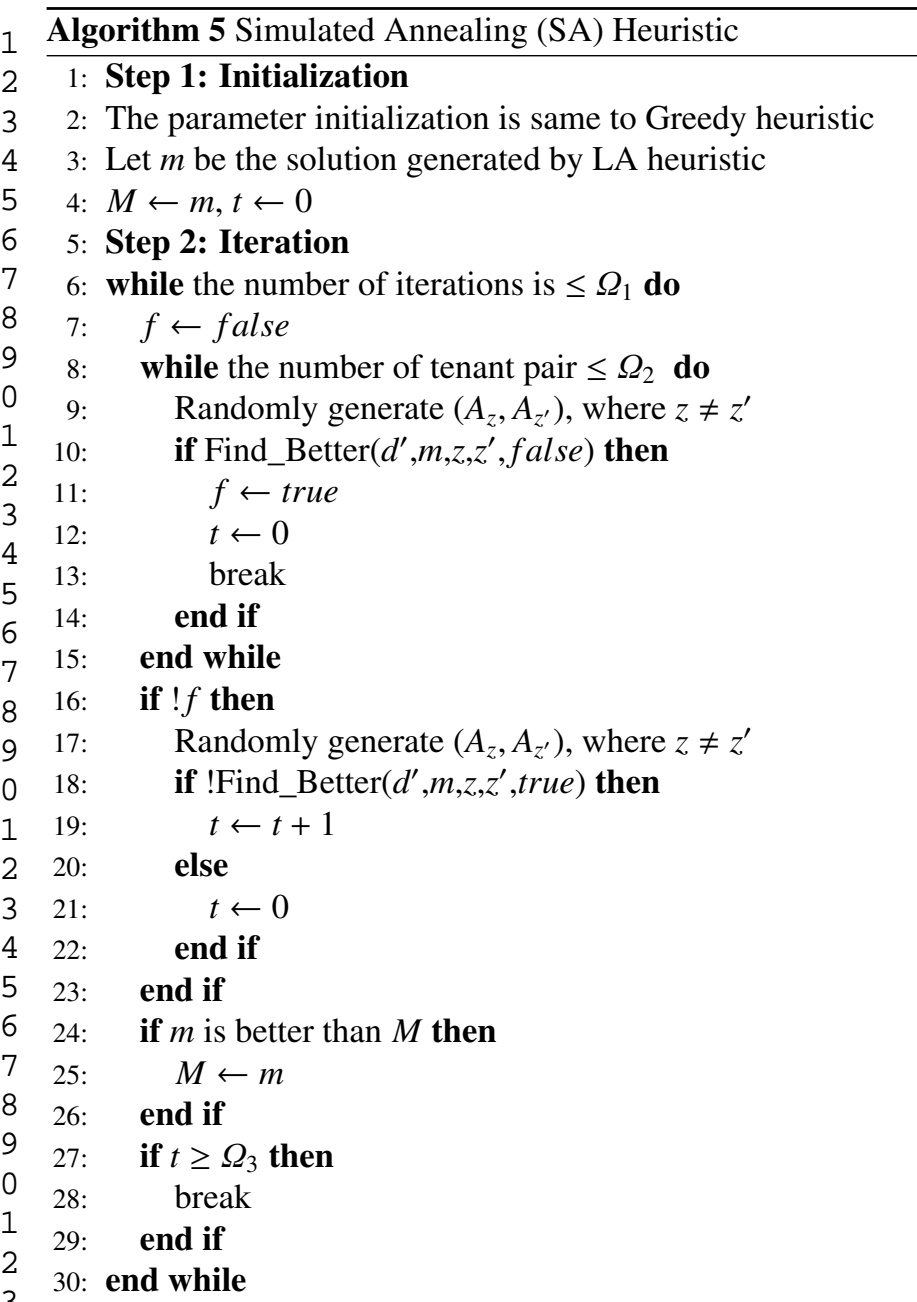

sult of SA heuristic is always better than that of LA heuristic, and then that of Greedy heuristic, while running time is on the contrary.

\section{Solutions When Lack of Resource}

In this section, we consider the assumption that there is not enough resource in cloud system to deploy all applications. To maximize the benefits for the cloud provider, the maximum number of deployed applications (MNA) needs to be insured, and the objectives of MML and MTL are also considered for the cloud provider, respectively.

\subsection{Problem Formulation}

In this case, each application may be deployed on cloud systems, it obeys the following constraints:

$$
\sum_{j: C_{j} \in C} \sum_{k: D_{k} \in D} x_{i j k} \leq 1, \quad \forall i: A_{i} \in A .
$$

The constraint MNA can be formulated as:

$$
x \in \underset{x^{\prime}}{\operatorname{argmax}} \sum_{i: A_{i} \in A} \sum_{j: C_{j} \in C} \sum_{k: D_{k} \in D} x_{i j k}^{\prime} \text {, subject to: (2)-(5), (8), }
$$

where $x$ is the three-dimensional vector of $x_{i j k}$ defined in Section 4.1. Thus, when we consider the objective of MML, the VM placement problem can be written as:

$$
\text { Minimize } \max _{i: A_{i} \in A} \sum_{j: C_{j} \in C} \sum_{k: D_{k} \in D} x_{i j k} d_{i j k}^{\prime}, \quad \text { subject to: (9). }
$$

Similarly, under the objective of MTL, the VM placement problem is:

$$
\min \sum_{i: A_{i} \in A} \sum_{j: C_{j} \in C} \sum_{k: D_{k} \in D} x_{i j k} d_{i j k}^{\prime}, \quad \text { subject to: (9). }
$$

\subsection{Algorithms Description}

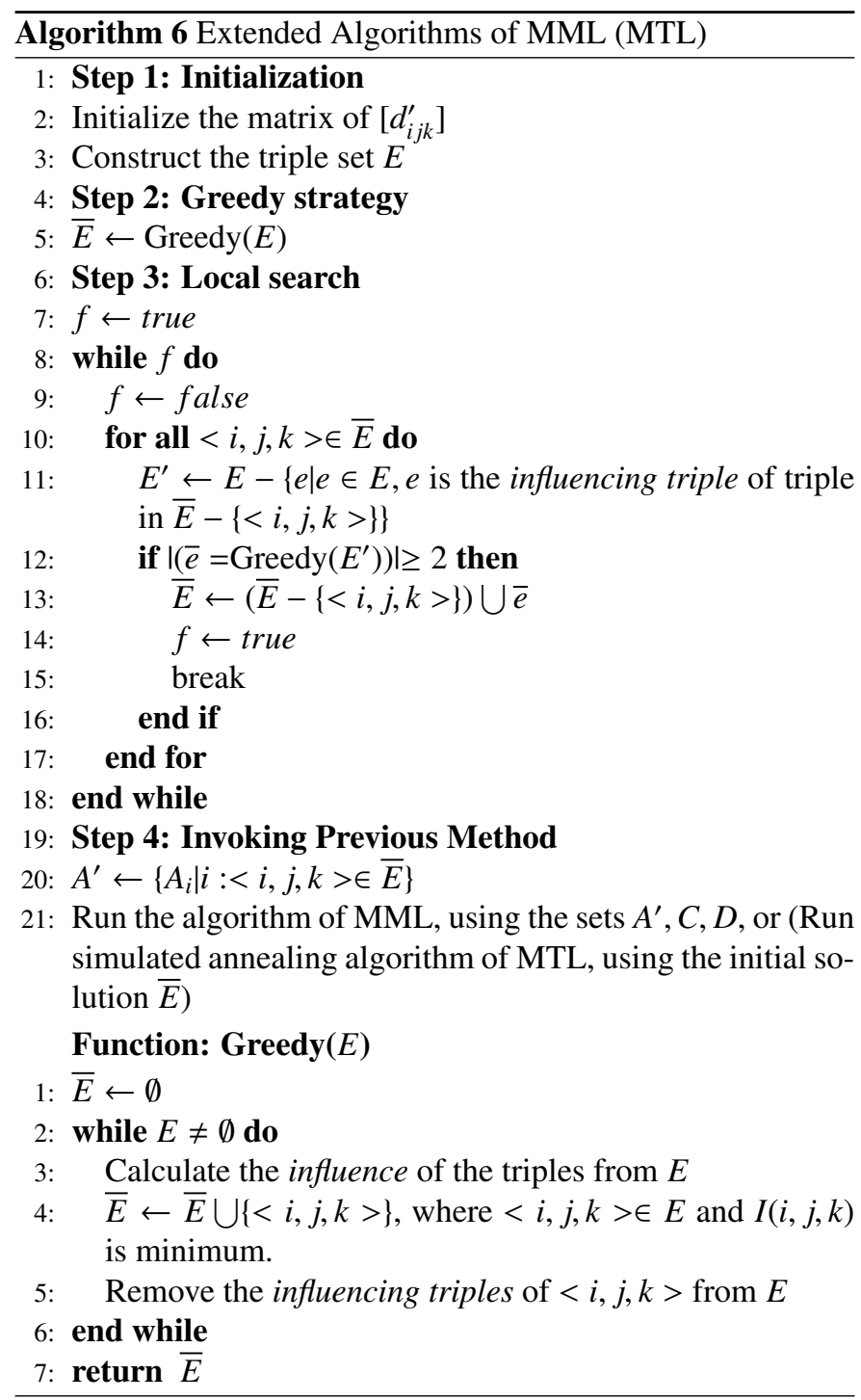

In this section, to solve this problem, we propose two extended heuristic algorithms, which are based on the thought of greedy and local search [30].

We first introduce the extended algorithm of MML. It mainly consists of four steps, initialization, greedy strategy, local search and invoking previous method, respectively. At the beginning, the triple set $E$ is obtained by the following rule: If 
and only if $d_{i j k}^{\prime} \leq \bar{W}_{i}$, the triple $<i, j, k>\in E$. Then, the process enter the step of greedy strategy. As shown in Section 5.2, the greedy strategy is always useful in solving the optimization problem in many situations. We can iteratively select appropriate triple from $E$. In order to find an appropriate triple, we make a following definition.

Definition 4. Assume $<i, j, k>\in E$, the influencing triple of $<i, j, k>i s<i^{\prime}, j^{\prime}, k^{\prime}>\in E$, which meet $i=i^{\prime}$ or $j=j^{\prime}$ or $k=k^{\prime} . I(i, j, k)$ is the influence of $\langle i, j, k\rangle$, which is the number of influencing triples of $\langle i, j, k\rangle$.

For example, we assume $E=\{<1,2,3>,<1,3,3>$ $,<2,3,1>$ \}, we can obtain that the influencing triples of $<1,2,3>$ are $<1,2,3>$ and $<1,3,3>$, so $I(1,2,3)=2$. Similarly, $I(1,3,3)=3$ and $I(2,3,1)=2$. In our greedy strategy, at each iteration, we select a triple from $E$, whose influence is minimum. Afterwards, we remove the influencing triples of it from $E$. When the set $E$ is empty, the iteration of greedy strategy is terminated. So far, a solution $\bar{E}$ of VM placement was obtained. In order to optimize this VM placement, we process the local search method from [30], which is based on the fac$t$ that removing one triple and adding two or more triples. At each iteration of local search, we find a triple $\langle i, j, k>\in \bar{E}$ so that when it is removed, we can add two or more triples into the solution $\bar{E}$. We say that such a triple is an adjustable triple. For any $<i, j, k>\in \bar{E}$, we obtain a triple set $E^{\prime}=E-\{e \mid e \in E, e$ is the influencing triple of triple in $\bar{E}-\{<i, j, k>\}\}$. It is obvious that all triples in $E^{\prime}$ are not the influencing triples of the triple in $\bar{E}-\{<i, j, k>\}$. Thus, we can add the triples from $E^{\prime}$ into $\bar{E}-\{<i, j, k>\}$ to update the solution $\bar{E}$. In our algorithm, we use the greedy strategy that is used at the second step, to obtain the triple set $\bar{e}$ from $E^{\prime}$. If $|\bar{e}| \geq 2$, the triple $<i, j, k>$ is an adjustable triple, and we can remove it and add the corresponding triples into $\bar{E}$, and the process enters the next iteration. If there does not exist adjustable triple, the iteration of local search is terminated, and the set $\bar{E}$ is a VM placement solution. So far, we used the heuristic method to obtain the number of deployed applications. From the literature [31], we can obtain that our method can reach the approximate performance of 2 on the number of deployed applications. Next, the algorithm enters the last step, invoking previous method. In this step, we run the previous algorithm to obtain the VM placement. Let $A^{\prime}$ denote the set of tenants accepted in $\bar{E}$. Then, the algorithm of MML is performed, using the sets $A^{\prime}, C, D$. Since $\bar{E}$ is a feasible VM placement, all of tenants in $A^{\prime}$ can be accepted. Thus, we can invoking the algorithm of MML to minimize the maximum latency for tenants $A^{\prime}$. The detailed algorithm description is shown in Alg. 6, and the greedy strategy subprocedure are listed at the bottom of the algorithm. Under the objective of MTL, we use the same method to obtain the number of deployed applications. Then, being similar with MML, the extended algorithm of MTL perform the simulated annealing algorithm of MTL using the initial solution $\bar{E}$ to minimize the total latency for the tenants $A^{\prime}$. The detail algorithm is also shown in Alg. 6, where the content in line 21 is in brackets.

\section{Numerical Results}

This section mainly presents the numerical results to demonstrate the efficiency of our algorithms. Then, we examine the impact of different parameters on the performance of our algorithms. We first introduce the simulation settings.

\subsection{Simulation Settings}

We take the experiments in Aliyun cloud platform [35], in which there are some data centers set up in different locations. To simulate our system model, we use some servers spreading over $K(K=1,2, \cdots, 8)$ data center(s). For simplicity, we assume the tenants' applications just simply access data from DNs. The average size for transmission data of each application $\alpha_{i}$ is generated randomly between $100 \mathrm{~KB}-200 \mathrm{~KB}$. In this case, these servers can deploy at most 500 applications and $500 \mathrm{DNs}$ at each data center. In our simulation, the required number of available VMs and DNs are randomly generated in $K$ data center(s). Since the number of VMs and the number of DNs in the same data center may be not equal, there may be some applications access data remotely. The data transmission speed between a VM and a DN that belong to the same data center is $100 \mathrm{MBps}$. When a VM and a DN are in the different data centers, the VM accesses data remotely, and the network bandwidth is $20 \mathrm{MBps}$. The tenants are simulated and randomly distributed around the data centers. The latency from a tenant to data centers are proportion to the distance between them. We assume the request rate $\left[\lambda_{i}\right]$ that from the tenant $A_{i}$ is randomly chosen from 30 to 80 , and the upper bound $\bar{W}_{i}$ for the response time is randomly chosen from 500 to 1000 (ms).

To examine our algorithms, we first perform them to obtain the VM placements, based on which, the VMs and the DNs are deployed on our data centers. Then, the tenants' requests are simulated to send to the corresponding VMs, and the performance is counted. We first take the experiments to show the maximum latency of the algorithm of MML and the average latency of the algorithms of MTL, respectively. Then, the performance of the extended algorithms is also examined. In our experiments, we observe the impact of different parameter$\mathrm{s}$, such as the number of tenants $l$, the number of VMs $m$ and the number of DNs $n$, etc., on the performance of the results of our algorithms. All results are obtained by averaging over 20 random system model instances.

\subsection{Simulation Results of $M M L$}

In this section, we evaluate the algorithm of MML. The effectiveness of decreasing the maximum response latency is first observed. Then, we examine the impact of the number of tenants, VMs and DNs on the results of the algorithm. In order to check the effectiveness of our algorithm, we compare it with the randomized approach, in which the VMs and DNs are assigned to the tenants randomly. In these experiments, we set the number of data centers $K=4$ to 8 , and the number of available VMs and DNs are both 2000 that randomly spread over $K$ data centers. To satisfy all tenants' requirements, we set the number of tenants is less than that of cloud resources (VM$\mathrm{s}$ and DNs), that is $l=1800$. The results are shown in Fig. 

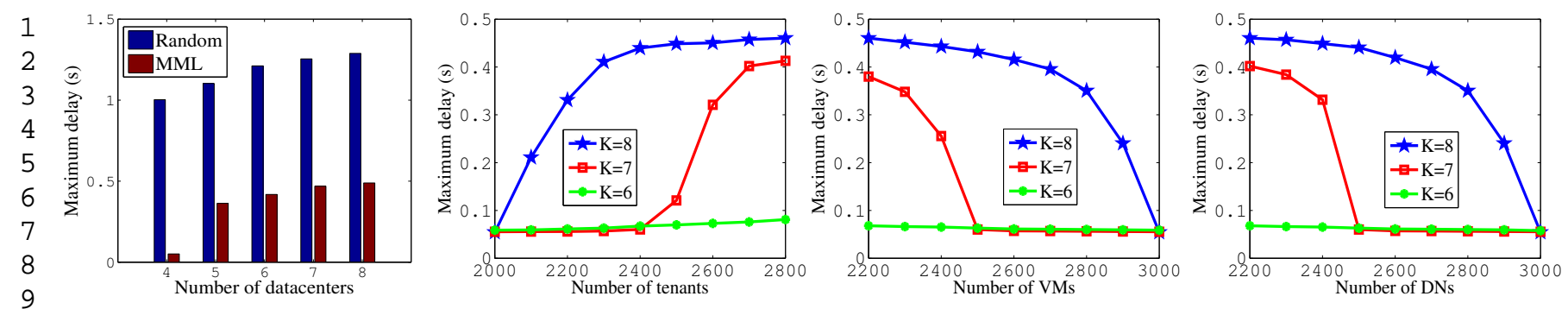

Figure 3: Maximum delay vs. NumberFigure 4: Maximum delay vs. NumberFigure 5: Maximum delay vs. Number Figure 6: Maximum delay vs. Number of datacenters of tenants of VMs of DNs
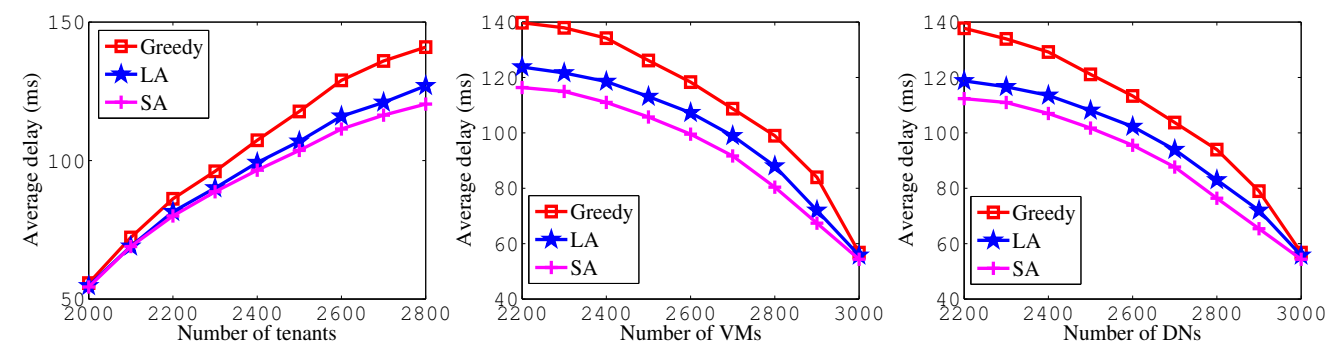

Figure 7: Average delay vs. Number ofFigure 8: Average delay vs. Number of datacenter
VMs of DNs
3 , where the red blocks represent the results of our algorithm, and the blue blocks represent the results of the randomized approach. We can see that our algorithm can effectively reduce the maximum response delay. For example, our algorithm can reduce the maximum response delay about $61 \%$ compared to randomized approach when there are 8 data centers. We also find that when there are 4 data centers, our algorithm reduces the maximum response delay about $94 \%$. This is because that the number of VMs and DNs are both 2000 and all of VMs and DNs in 4 data centers are available for deploying applications, thereby all applications access data locally. However, the randomized approach allocates resources randomly, leading to some applications accessing data remotely. Thus, the maximum response delay can be significantly reduced. Figure also shows that the maximum response delay grows as the number of data centers increases. This is because as the number of data centers increases, there will be more VMs and DNs that are not at the same location, leading to larger latency between them.

Then, we study the impacts of the number of tenants, VMs and DNs, which are shown in Fig. 4-6. Besides, the number of data centers is changed from 6 to $8(K=6,7,8)$. In these figures, the results of $K=6,7,8$ are represented by the green, red, blue curves respectively. We first observe the effect of the number of tenants that is varied from 2000 to 2800 . The number of VMs and DNs are both set to 3000. As shown in Fig. 4, the maximum response delay grows as the number of tenants increases. This is because as the number of tenants increases, there will be more competition of the cloud resources, leading to not best choices for some tenants. When there are 6 data centers, the growth of the maximum delay is not significant. This is because that in this scenario, the VM and the DN that are assigned to the same application are in the same data center, leading to accessing data locally. This reason also applies the case that there are 7 data centers and the number of tenants is less than 2500. Figure also shows that when the number of tenants is less than 2500, the maximum response delay of $K=7$ is less than that of $K=6$. The reason is that in these cases, all of applications access data locally and more data centers can decreases the distance between tenants and data centers, resulting in less latency.

The simulation results of the impact of the number of VMs from 2200 to 3000 are shown in Fig. 5, where the number of tenants is 2000 and the number of DNs is 3000 . The results of the impact of the number of DNs from 2200 to 3000 are shown in Fig. 6, where the number of tenants is 2000 and the number of VMs is 3000 . We can see that the maximum response delay trends down along with the number of VMs (DNs). This is because there are more choices of VMs (DNs) for tenants, and more VMs and DNs are at the same location, resulting in less service time, as the number of VMs (DNs) increases. Figures also show that the decrease of the maximum response delay is small when there are 6 data centers. The reason is the same as the one mentioned in previous experiments.

\subsection{Simulation Results of MTL}

In this section, using the parameter of average delay, we evaluate the algorithms of MTL, which includes Greedy, Local Adjustment (LA) and Simulated Annealing (SA). We first observe the performance of our algorithms by comparing them with the randomized approach, in which the resources are assigned to the tenants randomly, under the number of data centers from 4 to 8 . In this part of the simulations, the number of VMs and DNs are both 2000, and the number of tenants is 1800. The results are shown in Fig. 7. As it can be seen, the 

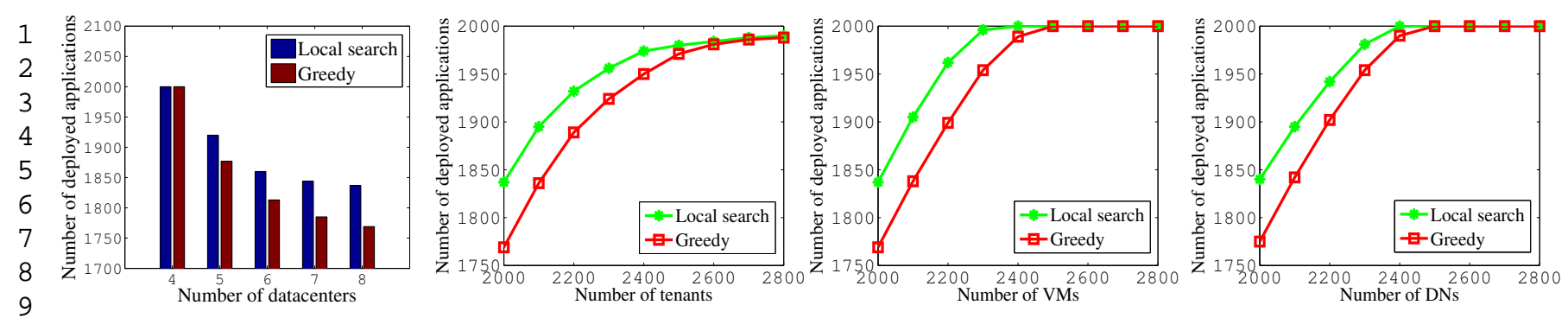

Figure 11: Number of deployed appli-Figure 12: Number of deployed appli-Fig cations vs. Number of datacenters cations vs. Number of tenants

average response delay increases along with the number of data centers. This is because as the number of data centers increases, there will be more VMs and DNs at different locations, and more applications will access data remotely, leading to larger link delay. Figure also shows that our algorithms can decrease much more average response delay of the requests compared with the randomized approach. For example, when there are 8 data centers, the SA heuristic achieves the best performance in our algorithms by decreasing the average response delay for about $69 \%$, while the LA heuristic and Greedy heuristic achieve the second and third optimization with the decreasing percentage as about $66 \%$ and $55 \%$ respectively.

Then, we study the impacts of the number of tenants, VMs and DNs, which are shown in Fig. 8-10, where the number of data centers is set to 8 . In each figure, the red curve represents the results of Greedy heuristic, while the blue curve represents the results of LA heuristic. The performance of SA heuristic is illustrated by the purple curve. From all of these figures, we can see that the SA heuristic outperforms other approaches in all scenarios, and the performance of LA heuristic is better than that of Greedy heuristic. The average response delay of SA heuristic decreases about $20 \%$ of the Greedy increase on average, while the LA heuristic deceases the average response delay for about $17 \%$ on average.

The simulation results with the number of tenants from 2000 to 2800 are shown in Fig. 8, where the number of VMs and DNs are both 3000 . The average response delay increases as number of tenants becomes larger. The reason is that when the number of tenants becomes larger, more resources are needed, which results in the increase of number of waiting queues and more average response delay. When there are 2000 tenants, our algorithms nearly get the same average response delay. The reason is that all applications access data locally, thereby the SA algorithm cannot have a significant improvement on decreasing latency.

The simulation results with the number of VMs from 2200 to 3000 and are shown in Fig. 9, where the number of tenants is 2000 and the number of DNs is 3000 , and that with the number of DNs from 2200 to 3000 are shown in Fig. 10, where the number of tenants is 2000 and the number of VMs is 3000 . We can see that the average response delay of the requests trends down along with the number of VMs (DNs). This is because there will be more choices available for tenants, and more DNs (VMs) will get closer to the VMs (DNs) as the number of VMs
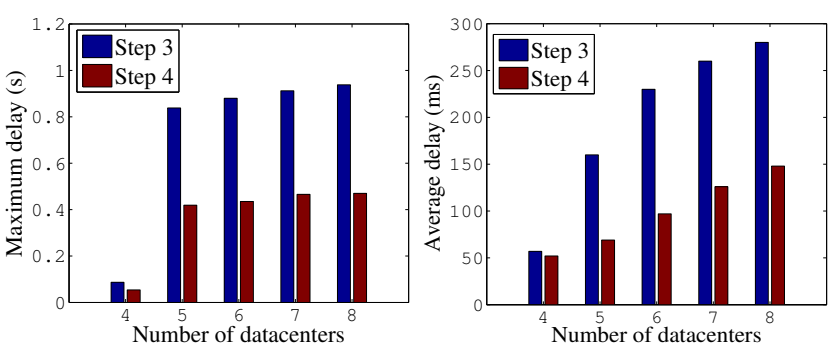

Figure 15: Maximum delay vs. Num-Figure 16: Average delay vs. Number ber of datacenters of datacenters

(DNs) increases.

\subsection{Simulation Results of Extended Algorithms}

In this section, we evaluate the extended algorithms. Since two extended algorithms have the same process of obtaining the maximum number of deployed applications, we first observe the number of deployed applications of our algorithms. We change the number of data centers from 4 to 8 and compare the results of our algorithms, which are obtained by the third step (Local search) in Alg. 6, with that of the greedy strategy, which are obtained by the second step (Greedy strategy). The number of VMs and DNs are both 2000 and the number of tenants is 1800, and the results are shown in Fig. 11, where the blue blocks represent the results of our algorithms while the red blocks represent the results of greedy strategy. We can find that the number of deployed applications decreases along with the number of data centers. This is because there will be more VMs and DNs that are in different locations, leading to larger latency between them. Figure also shows that the results of our algorithms are larger than that of the greedy strategy.

Next, we study the impacts of the number of tenants, VMs and DNs. The results are shown in Fig. 12-14, where the green and red curves represent the results of our algorithms and the greedy strategy respectively. There are 8 data centers in these experiments. We first observe the impact of the number of tenants. Here we varied the number of tenants from 2000 to 2800 , and the number of VMs and DNs are both fixed to 2000. From Fig. 12, it can be seen that the number of deployed application$\mathrm{s}$ increases along with the number of tenants. This is because there will be more tenants that get the chance to be deployed on cloud systems. The figure also shows that when the number of tenants is larger than 2600 the results of our algorithms is 
close to that of the greedy strategy. This is because that there are several VMs and DNs in poor transmission conditions.

The simulation results with the number of VMs from 2000 to 2800 are shown in Fig. 13, where the number of tenants and DNs are both 2000, and that with the number of DNs from 2000 to 2800 are shown in Fig. 14, where the number of tenants and VMs are both 2000. We can see that the number of deployed applications grows as number of VMs (DNs) increases. This is because as the number of VMs (DNs) increases, there will be more VMs (DNs) closer to the DNs (VMs) and tenants, leading to less service time between them. The tenants will also be able to choose from more VMs (DNs). Figure also shows that when the number of VMs (DNs) is larger than 2500, our data centers can deploy all applications.

Finally, The maximum response delay, which is the result of the extended algorithm of MML, and the average response delay, which is the result of the extended algorithm of MTL, are examined respectively. We compare the results of the extended algorithms (step 4 in Alg. 6) with that of the local search method (step 3 in Alg. 6). The number of data centers is varied from 4 to 8 , and the number of VMs, DNs and tenants are all equal to 2000. The results of the maximum response delay are shown in Fig. 15, where the blue blocks represent the results of the local search method, while the red blocks represent the results of the extended algorithm. As it can be seen, the extended algorithm can effective decrease the maximum delay. Figure also shows that the maximum response delay grows as the number data centers increases. The reason is the same as the one that mentioned in previous experiments. The results of the average response delay are shown in Fig. 16. From this figure, we can see our extended algorithm significantly decreases the average response delay. The average response delay grows as the number data centers increases. The reason is also due to more VMs and DNs being in different locations.

\section{Conclusion}

In this paper, we study the techniques of VM placement in distributed cloud systems with stochastic requests from the tenants. We model the requests of each application from corresponding tenant as independent Poisson stream. In addition, the VMs with their data nodes are modeled as simple M/M/1 queueing systems, that is, the service time at each VM obeys the exponential distribution. We define VM placement problem with two objectives including MML and MTL. For the objective of MML, we propose an approximate algorithm, which can reach the approximate performance of $1+\varepsilon$. Then, three heuristic algorithms are proposed to solve the problem with MTL. Considering the condition of lacking of resource, we maximize the number of deployed applications, and two extended algorithms of MML and MTL are proposed respectively. According to simulation results, our algorithms are efficient in their corresponding objectives [8].

\section{Acknowledgment}

This paper is supported by the National Science Foundation of China under No. U1301256, 61170058, and 61472383, Special Project on IoT of China NDRC (2012-2766), Research Fund for the Doctoral Program of Higher Education of China No. 20123402110019, the Natural Science Foundation of Anhui Province in China under No. 1408085MKL08.

\section{References}

[1] M. Randles, D. Lamb, E. Odat, A. Taleb-Bendiab, Distributed redundancy and robustness in complex systems, Journal of Computer and System Sciences 77 (2) (2011) 293-304.

[2] Amazon ec2, http://aws. amazon.com/ec2.

[3] Cisco data center, http://goo.gl/Sil548.

[4] C. Hyser, B. Mckee, R. Gardner, B. J. Watson, Autonomic virtual machine placement in the data center, Hewlett Packard Laboratories, Tech. Rep. HPL-2007-189 (2007) 2007-189.

[5] X. Li, Z. Qian, S. Lu, J. Wu, Energy efficient virtual machine placement algorithm with balanced and improved resource utilization in a data center, Mathematical and Computer Modelling 58 (5) (2013) 1222-1235.

[6] O. Biran, A. Corradi, M. Fanelli, L. Foschini, A. Nus, D. Raz, E. Silvera, A stable network-aware vm placement for cloud systems, in: Proceedings of the 2012 12th IEEE/ACM International Symposium on Cluster, Cloud and Grid Computing (ccgrid 2012), IEEE Computer Society, 2012, pp. 498-506.

[7] H. Teyeb, A. Balma, N. B. Hadj-Alouane, S. Tata, Optimal virtual machine placement in a multi-tenant cloud, in: Service-Oriented ComputingICSOC 2014 Workshops, Springer, 2015, pp. 308-319.

[8] M. Alicherry, T. Lakshman, Optimizing data access latencies in cloud systems by intelligent virtual machine placement, in: INFOCOM, 2013 Proceedings IEEE, IEEE, 2013, pp. 647-655.

[9] M. Johnston, H.-W. Lee, E. Modiano, Robust network design for stochastic traffic demands, Journal of Lightwave Technology 31 (18) (2013) 3104-3116.

[10] Apache hadoop, http://hadoop.apache.org.

[11] R. Cohen, L. Lewin-Eytan, J. S. Naor, D. Raz, Almost optimal virtual machine placement for traffic intense data centers, in: INFOCOM, 2013 Proceedings IEEE, IEEE, 2013, pp. 355-359.

[12] W. Huang, X. Li, Z. Qian, An energy efficient virtual machine placement algorithm with balanced resource utilization, in: Innovative Mobile and Internet Services in Ubiquitous Computing (IMIS), 2013 Seventh International Conference on, IEEE, 2013, pp. 313-319.

[13] Y. Gao, H. Guan, Z. Qi, Y. Hou, L. Liu, A multi-objective ant colony system algorithm for virtual machine placement in cloud computing, Journal of Computer and System Sciences 79 (8) (2013) 1230-1242.

[14] W. Fang, X. Liang, S. Li, L. Chiaraviglio, N. Xiong, Vmplanner: Optimizing virtual machine placement and traffic flow routing to reduce network power costs in cloud data centers, Computer Networks 57 (1) (2013) 179-196.

[15] J. Dong, X. Jin, H. Wang, Y. Li, P. Zhang, S. Cheng, Energy-saving virtual machine placement in cloud data centers, in: Cluster, Cloud and Grid Computing (CCGrid), 2013 13th IEEE/ACM International Symposium on, IEEE, 2013, pp. 618-624.

[16] J. T. Piao, J. Yan, A network-aware virtual machine placement and migration approach in cloud computing, in: 2010 Ninth International Conference on Grid and Cloud Computing, IEEE, 2010, pp. 87-92.

[17] H. T. Vu, S. Hwang, A traffic and power-aware algorithm for virtual machine placement in cloud data center, International Journal of Grid \& Distributed Computing 7 (1) (2014) 350-355.

[18] K. Li, H. Zheng, J. Wu, Migration-based virtual machine placement in cloud systems, in: Cloud Networking (CloudNet), 2013 IEEE 2nd International Conference on, IEEE, 2013, pp. 83-90.

[19] X. Li, J. Wu, S. Tang, S. Lu, Let's stay together: Towards traffic aware virtual machine placement in data centers, in: IEEE INFOCOM 2014IEEE Conference on Computer Communications, IEEE, 2014, pp. 18421850 . 
20] J. W. Jiang, T. Lan, S. Ha, M. Chen, M. Chiang, Joint vm placement and routing for data center traffic engineering, in: INFOCOM, 2012 Proceedings IEEE, IEEE, 2012, pp. 2876-2880.

[21] A. Amokrane, M. F. Zhani, R. Langar, R. Boutaba, G. Pujolle, Greenhead: Virtual data center embedding across distributed infrastructures, IEEE Transactions on Cloud Computing 1 (1) (2013) 36-49.

[22] Y. Xia, M. Zhou, X. Luo, S. Pang, Q. Zhu, Stochastic modeling and performance analysis of migration-enabled and error-prone clouds, IEEE Transactions on Industrial Informatics 11 (2) (2015) 495-504.

[23] M. Malekimajd, A. Movaghar, S. Hosseinimotlagh, Minimizing latency in geo-distributed clouds, The Journal of Supercomputing 71 (12) (2015) 4423-4445.

[24] C.-N. Mao, M.-H. Huang, S. Padhy, S.-T. Wang, W.-C. Chung, Y.-C. Chung, C.-H. Hsu, Minimizing latency of real-time container cloud for software radio access networks, in: 2015 IEEE 7th International Conference on Cloud Computing Technology and Science (CloudCom), IEEE, 2015, pp. 611-616.

[25] K. Jang, J. Sherry, H. Ballani, T. Moncaster, Silo: predictable message latency in the cloud, ACM SIGCOMM Computer Communication Review 45 (4) (2015) 435-448.

[26] Q. Pu, G. Ananthanarayanan, P. Bodik, S. Kandula, A. Akella, P. Bahl, I. Stoica, Low latency geo-distributed data analytics, ACM SIGCOMM Computer Communication Review 45 (4) (2015) 421-434.

[27] L. Zhang, Z. Li, C. Wu, Dynamic resource provisioning in cloud computing: A randomized auction approach, in: IEEE INFOCOM 2014-IEEE Conference on Computer Communications, IEEE, 2014, pp. 433-441.

[28] D. Gross, Fundamentals of queueing theory, John Wiley \& Sons, 2008.

[29] V. Kann, Maximum bounded 3-dimensional matching is max snpcomplete, Information Processing Letters 37 (1) (1991) 27-35.

[30] C. A. J. Hurkens, A. Schrijver, On the size of systems of sets every t of which have an sdr, with an application to the worst-case ratio of heuristics for packing problems, SIAM Journal on Discrete Mathematics 2 (1) (1989) 68-72.

[31] M. Cygan, Improved approximation for 3-dimensional matching via bounded pathwidth local search, in: Foundations of Computer Science (FOCS), 2013 IEEE 54th Annual Symposium on, IEEE, 2013, pp. 509518.

[32] H. W. Kuhn, The hungarian method for the assignment problem, Naval research logistics quarterly 2 (1-2) (1955) 83-97.

[33] J. A. Bondy, U. S. R. Murty, Graph theory with applications, Vol. 290, Citeseer, 1976.

[34] R. E. Burkard, E. Cela, Linear assignment problems and extensions, in: Handbook of combinatorial optimization, Springer, 1999, pp. 75-149.

[35] Aliyun, http://www.aliyun.com/. 